\title{
GW23-e0242 PREVALENCE AND TRENDS IN HEART DISEASES DURING THE PAST 8 YEARS (2004-2011) IN CHINA BASED ON AN ECHOCARDIOGRAPHY DATA
}

doi:10.1136/heartjnl-2012-302920d.25

${ }^{1}$ Wenzhi Pan, ${ }^{2}$ Leiei Cheng, ${ }^{2}$ Xianhong Shu, ${ }^{1} J u n b o$ Ge. ${ }^{1}$ Department of Cardiology, Shanghai Institute of Cardiovascular Diseases, Zhongshan Hospital, Fudan University, Shanghai, China; ${ }^{2}$ Department of Echocardiograph, Shanghai Institute of Cardiovascular Diseases, Zhongshan Hospital, Fudan University, Shanghai, China

Objectives Data about prevalence and trends in heart diseases during the recent years in China are lacking. Using a large echocardiography data in our center, the objective of the study was to analyse the prevalence and trends in several common heart diseases during the past 8 years.

Methods This study retrospectively analysed the 2-D echocardiographic data in our department from 2004 to 2011.

Results A total of 312850 cases were included in the study. There was a trend toward decreasing incidence of rheumatic heart disease during past 8 years, from $5.49 \%$ in 2004 to $3.05 \%$ in 2011. Infective endocarditis was also decreased, from an average incidence of $0.37 \%$ during the first 4 years to $0.27 \%$ during the last 4 years. The incidence of hypertrophic cardiomyopathy, including 20\% apical hypertrophic cardiomyopathy and 20\% hypertrophic obstructive cardiomyopathy, was about $1.8 \%$. The total incidence of three most common congenital heart diseases, that is, atrial septal defect, ventricular septal defect and patent ductus arteriosus, decreased about 30\% during the 8 years, from 5.53\% in 2004 to $3.80 \%$ in 2011 . The incidence of patients with moderate pulmonary arterial hypertension (PAH) or left ventricular systolic dysfunction (LVSD) decreased during the 8 years, while severe $\mathrm{PAH}$ or LVSD did not change.

Conclusions The present study showed the prevalence and trends in several common heart diseases during the past 8 years in China. 